\title{
Erstellung eines Geschäftsmodells zur Ausrüstung von Taxi Berlin mit einem digitalen Bündelfunksystem
}

\author{
Bernd Eylert, Patrik Groh, Eike Kräft, Thomas Weidner
}

\section{Kurzfassung}

Taxi Berlin hatte im Jahr 2005 eine Ausschreibung herausgebracht mit dem Ziel, ihr analoges Bündelfunksystem in ein digitales umzuwandeln. Daran beteiligen wollte sich auch die Firma adKor GmbH, ein KMU (kleine und mittlere Unternehmen) aus Wildau. Es bat die TFH Wildau um Unterstützung. Aufgabe der Hochschule war nun, ein komplettes Geschäftsmodell für diesen Fall zu entwickeln. Gleichzeitig sollte es so allgemein formuliert und gerechnet sein, dass man es für ähnliche Ausschreibungen in anderen Bundesländern wieder verwenden kann. Wettbewerbssituation des Unternehmens, Markt und Technologie wurden analysiert, ein Marketingkonzept entwickelt und verschiedene Szenarien beschrieben und gerechnet. Abschließend wurden Handlungsempfehlungen gegeben. Obwohl das Konzept in Berlin nicht zum Tragen kam, war es später erfolgreich bei der Bewerbung von adkor zum Erwerb der Bündelfunksysteme in Hamburg und Hannover und hat so auch seinen Praxistest bestanden.

\begin{abstract}
In 2005, Taxi Berlin offered a call for tenders to switch their trunking system from analogue to digital. A small and medium enterprise (SME) in Wildau, adKor GmbH, wanted to participate in the contest and asked the local university for support. The task was to write a business model and calculate a business case for Taxi Berlin, but keep it open and write it generally so that the client could use it for other tenders for switching from analogue to digital trunking systems in other parts of Germany as well. Competition, market and technology were analysed, a marketing concept developed and different scenarios described and calculated. Finally, a recommended course of action was given. Although the business model was not successful in Berlin, it succeeded in Hamburg and Hannover.
\end{abstract}

\section{Einleitung}

Teil der Lehrveranstaltung »Mobile Commerce «im Masterstudiengang Telematik ist die Durchführung eines Projekts, in dem die Studierenden das Gelernte praktisch erproben sollen. Um dem Anspruch an Masterstudenten gerecht zu werden, soll es sich möglichst um ein reales Thema handeln. In diesem Rahmen ergab sich Ende 2005 ein kleiner Forschungsauftrag bei der Wildauer adKor GmbH zum Thema »Erstellung eines Geschäftsmodells zur Ausrüstung von Taxi Berlin mit neuem digitalem Bündelfunk «, mit dem sich die Firma kurzfristig an der Ausschreibung von Taxi Berlin bewerben wollte. Das entwickelte Modell sollte aber allgemeingültig aufgesetzt werden, damit sich das Unternehmen auch an anderen Stellen mit diesem Geschäftsmodell um den Erwerb digitaler Bündelfunknetze bewerben kann. Das Modell wurde aus Wettbewerbsgründen mit realistischen, aber synthetischen Kennzahlen und Preisangaben für die Erstellung der Finanzierungsszenarien durchgeführt.

\section{Aufgabenstellung}

Ziel der Arbeit ist die Erstellung eines Geschäftsmodells für die Ausrüstung von Taxi Berlin mit einem digitalen Bündelfunksystem. Dies geschieht im Auftrag der Firma adKor GmbH, die gegenüber Taxi Berlin als Auftragnehmer auftritt.

Laut Vorgabe von adKor sollen mehrere Szenarien erstellt und gerechnet werden. Diese beinhalten i. W. verschiedene Möglichkeiten der Vertragsmodalitäten zwischen adKor und Taxi Berlin im Bereich der Nutzung der aufzubauenden Netzinfrastruktur und der auszustattenden Funkzentrale sowie der Wieder-/Weiterverwendbarkeit einschl. Verkauf der existierenden Technik.

Dazu soll das Modell unter den Gesichtspunkten »Unternehmen und ihre Leistungsfähigkeit«, »Technologie«, »Märkte und Marketing « sowie »Finanzierung/ Investition« betrachtet werden. Ein weiteres Ziel dieser Maßgaben ist die Abstrahierung des individuellen Geschäftsmodells zwischen adKor und Taxi Berlin, um aus diesen Betrachtungen einen Mehrwert für den Auftraggeber zu generieren, damit er mit diesem Geschäftsmodell auch in anderen Städten und Regionen in den Wettbewerb um die Errichtung digitaler Bündelfunksysteme für öffentliche Auftraggeber eingreifen kann. 


\section{Unternehmen}

Dieses Kapitel beschreibt die Firmen näher, für die die Erstellung des Geschäftsmodells relevant sein soll, und zeigt auf, welche unterschiedlichen Ansprüche die beiden Unternehmen an den Inhalt des Geschäftsmodells stellen.

\subsection{Taxi Berlin}

Die Firma Taxi Berlin ist in Berlin ein Anbieter von Taxifunk und betreibt ein eigenes computergestütztes Vermittlungssystem, über das die Taxen ihre Fahraufträge erhalten. Durch den digitalen Datenfunk können auch GPS-Empfänger in die Taxis eingebaut werden, die dann ihre Positionsdaten an die Zentrale übermitteln. So kann auf einem digitalen Stadtplan die Position der Fahrzeuge angezeigt werden. Nach den Vorstellungen von Taxi Berlin sollen in die Fahrzeuge, wenn nicht schon geschehen, Bildschirme eingebaut werden, auf denen die Fahrer die Informationen des nächsten Fahrauftrags angezeigt bekommen. Durch die jeweils aktuelle Positionsbestimmung der Fahrzeuge soll die Disponierung in der Zentrale effizienter gestaltet werden.

Die Anforderung seitens Taxi Berlin an die Lösung ist eindeutig: Für möglichst wenig Geld soll das neue System inklusive einer neuen Zentrale eingeführt werden. $\mathrm{Zu}$ den Zahlungsmodalitäten gab es keine Vorgaben. Das wird Bestandteil der Szenarien sein, die im Rahmen des Projektes erstellen werden.

\section{2 adKor $\mathrm{GmbH}$}

Die Firma adKor beschäftigt sich mit Planung, Beratung und Service im Bereich Telekommunikation. Dazu zählen Wireless LAN, Mobilfunk und Festnetz. Dabei geht es um die Akquisition, Planung und Aufbau von Antennensystemen und Infrastruktur für deren Betrieb. Obwohl das Unternehmen selbst sehr jung ist, haben seine Mitarbeiter langjährige Erfahrung auf den angesprochenen Gebieten. Taxi Berlin trat an adKor heran, um ein Geschäftsmodell für die Umstellung und den Betrieb von digitalem Bündelfunk zu entwickeln. adKors Strategie besteht darin, sich mit einem flexiblen Geschäftsmodell und mehreren Szenarien zu bewerben, in der Hoffnung, dass eines der beschriebenen und gerechneten Szenarien bei Taxi Berlin auf Zustimmung stößt.

\section{Technologie}

Dieses Kapitel stellt die eingesetzte Technik des Projektes vor. Es soll einen Überblick geben, warum gerade diese Technologie gewählt wurde, woraus die Infrastruktur besteht und wie sie sich zusammensetzt. Es soll auch eine Übersicht über die benötigte Hardware geben. Auf dieser Grundlage findet die Finanzierungsplanung für das Geschäftsmodell statt.

\subsection{Bündelfunk}

Beim Taxifunk handelt es sich um ein Bündelfunksystem. Bündelfunk erlaubt die Übertragung von Daten und Sprache in geschlossenen Benutzergruppen. Dabei werden mehrere Kanäle zusammengefasst und dynamisch zugewiesen, so dass eine höhere Verfügbarkeit erreicht wird. Im analogen Bündelfunk wird dabei ein Frequenzbereich zwischen 410 und $430 \mathrm{MHz}$ genutzt. Der Uplink erfolgt im Bereich zwischen 410 und 420 $\mathrm{MHz}$ und der Downlink findet im Bereich zwischen 420 und $430 \mathrm{MHz}$ statt. Die Datenübertragung erfolgt dabei mit nur $1,2 \mathrm{kbit} / \mathrm{s}$.

Im Fall von Taxifunk Berlin werden die Fahrzieldaten in Datenform auf ein grafisches Display im Fahrzeug übertragen. Meldungen der Fahrer kommen nach wie vor via Sprache ins System. Im analogen Bündelfunk können die Daten auf drei Arten übertragen werden:

- Im Organisationskanal als SDM (Short Data Message), 184 Bit oder EDM (Extended Data Message) mit maximal 100 Zeichen.

- Statusmeldungen von 5 Bit Länge (32 mögliche Meldungen).

- Datenübertragung im Sprachkanal mit Modem oder Akustikkoppler.

Wie überall findet auch auf dem Gebiet des Bündelfunks ein Generationswechsel statt - vom analogen hin zum digitalen Bündelfunk.

Es gibt dabei zwei mögliche Technologien: TETRA (TErrestrial Trunked RAdio) und TETRAPOL. Die beiden Technologien sind direkt zueinander inkompatibel. Sie können jedoch über Bridges verbunden werden. Abgesehen von der Technologie liegt der Hauptunterschied vor allem in den Zielmärkten. TETRAPOL wird in Frankreich bei Polizei und Gendamerie, in Deutschland oft für Funknetze im Bereich von Flughäfen eingesetzt. TETRA hingegen wird vor allem im öffentlichen Bündelfunk angewandt. Die beiden Technologien ergänzen sich also eher, als dass sie in Konkurrenz zueinander stehen.

In unserem Projekt hat sich die Firma Taxi Berlin für das TETRA-System entschieden. Das hat zur Folge, dass in alle Taxis neue Funkgeräte eingebaut werden müssen. Dies stellt einen hohen Kostenfaktor für die Projektplanung und -finanzierung dar.

\subsection{TETRA}

Die Technik, die für die Realisierung eines digitalen Bündelfunksystems genutzt werden soll, ist TETRA. Es handelt sich um einen ETSI-konformen Mobilkommunikationsstandard (Quelle: www.etsi.org). Vorteile gegenüber dem analogen Bündelfunk bestehen in der Möglichkeit der Verschlüsselung, so dass geschlossene Nutzergruppen auch wirklich unter sich bleiben, und der Möglichkeit, ein »Full Duplex« Funkgespräch zu führen, das heißt zwei Teilnehmer sprechen gleichzeitig miteinander und verstehen sich auch. Der analoge Bündelfunk verfügt nur über Halb-Duplex Möglichkeiten. Pro TETRA Kanal steht eine Nutzübertragungskapazität von 7,2 kBit/s zur Verfügung. Das erscheint nicht viel, allerdings kann die Kapazität durch die Bündelung von 
Kanälen auf 28,8 kbit/s gesteigert werden. Um auch für künftige Anwendungen mit gesteigerten Datenraten gerüstet zu sein, sorgt TEDS (TETRA Enhanced Data Service) für eine Übertragungsrate von bis zu $400 \mathrm{kBit} / \mathrm{s}$. So werden in Zukunft auch Videoübertragungen möglich sein. TETRA arbeitet mit einem Zeitmultiplexsystem mit vier unabhängigen Kanälen pro Träger. Der Trägerabstand beträgt $25 \mathrm{kHz}$, was gegenüber dem analogen Bündelfunk eine bessere Frequenzausnutzung bedeutet. Für das Gegensprechen wird bei TETRA das Time Division Duplex-Verfahren angewandt. Als Frequenzbereiche für die zivile Nutzung kommen grundsätzlich die Bereiche 285-290 MHz, 395-399,9 MHz, 410-430 MHz, 450-470 $\mathrm{MHz}, 870-876 \mathrm{MHz}$ und 915-921 MHz in Frage, wobei der 410-430 MHz-Bereich Priorität hat.

Taxi Berlin wird in erster Line die Datenübertragung für die Zuteilung von Fahraufträgen an die Fahrer nutzen. Diese haben in den Taxis Datendisplays, auf denen das Ziel mit anderen Angaben wie Name, Fahrziel, Behinderung usw. angegeben wird. Der Sprechfunk kann für die Kommunikation unter den Fahrern genutzt werden. Um die Disponierung der Fahrer zu erleichtern, sollen künftig in die Fahrzeuge GPS-Empfänger eingebaut werden. Diese übermitteln die Positionsdaten an die Zentrale, von wo das nächstgelegene Fahrzeug zum Kunden geschickt wird. Sollte TEDS eingeführt und kompatibel zu den installierten Funkgeräten sein, können auch im Notfall per eingebauter Videokameras Bildsequenzen übertragen werden. Es ist ebenfalls an den Anschluss des Taxi TETRA Funks an das Internet zu denken. So könnten elektronische Zahlungen sofort durchgeführt werden.

\subsection{Netzplan (Beispiel)}

Um ein digitales Funknetz mit einer Abdeckung für Berlin und das nahe Umland zu gewährleisten, sind umfangreiche Installationen im Raum Berlin zu tätigen. Dazu zählen die Installation und Einrichtung der Zentrale, aber vor allem das Aufstellen von ca. 4 bis 6 Basisstationen sowie deren Verbindung untereinander und mit der Zentrale mittels Richtfunkverbindung und Standleitungen. Dabei müssen nicht alle Basisstationen (BS) mit der Zentrale verbunden werden. Es reicht, wenn die Hälfte der BS mit der Zentrale direkt über Standleitungen verbunden ist, die anderen BS werden dann über Richtfunklink ins Netz eingebunden. Auf der anderen Seite steigt mit jeder direkt mit der Zentrale verbundenen BS die Ausfallsicherheit. So ist letztlich zwischen der Kosteneinsparung bei den Verbindungen und der Ausfallsicherheit des Netzes abzuwägen.

Die Standorte für die BS werden voraussichtlich der Müggelturm, die Pyramide, der Fernsehturm, der Schäferberg und ein $300 \mathrm{~m}$ hoher Mast in Hennigsdorf sein. Das waren also 5 Funkzellen. Die Standorte ergeben für Berlin und das nahe Umland eine ausreichende Funknetzabdeckung sowie Reserven, sollte eine Station innerorts ausfallen.

Die Anzahl und Platzierung der BS wurde beispielhaft von adKor vorgeben, Projekt und Vorplanungen näher erläutert.

\subsection{Komponenten}

Hier folgt eine Aufstellung der für die Netzinfrastruktur benötigten technischen Komponenten. Der Einfachheit halber werden die Komponenten für die BS einmalig behandelt, dazu kommen die Komponenten für den Richtfunk und die Verbindung mit der Taxifunkzentrale.

\subsubsection{Funkstandorte}

Zu einer Basisstation gehören mindestens eine Sendeantenne, in unserem Fall ein $120^{\circ}$-Strahler, die zugehörige Steuerungstechnik sowie die Verbindung zur Zentrale in Form einer Richtfunkstrecke (2 MBit) und/oder einer 2 bzw. 4 MBit-Standleitung.

Zu einem Richtfunklink gehören immer zwei Antennen samt zugehöriger Steuertechnik. Für die Richtfunkantennen kommen Antennendurchmesser von 60 und $120 \mathrm{~cm}$ in Betracht. Je weiter die Stationen voneinander entfernt sind, desto größer muss der Antennendurchmesser sein, um die gewünschte Datenrate von $2 \mathrm{MBit}$ zu gewährleisten. Die Komponenten umfassen für einen Richtfunklink eine Outdoor-Unit mit Radio, Sender/ Empfänger sowie Antenne. Dazu kommt eine IndoorUnit, die die 2 MBit-Schnittstelle umfasst.

Die Sendemasten, die sich in der Stadt befinden, benötigen pro Mast drei $120^{\circ}$-Antennen, um eine Abdeckung von $360^{\circ} \mathrm{zu}$ erreichen. Außerorts reichen zwei $120^{\circ}$-Strahler, um die Lücke zwischen Stadt und Umland zu schließen. Ein weiterer Grund für die begrenzte Ausleuchtung sind die Grenzen des von der Bundesnetzagentur zur Verfügung gestellten Funkbereichs. Ab einer bestimmten geografischen Grenze darf der Empfang einen bestimmten dB-Betrag nicht überschreiten. So werden Überschneidungen mit anderen Funkbereichen vermieden.

Für die Verbindung zwischen der Zentrale und den Funkmasten kommen grundsätzlich zwei Verbindungsarten in Frage: die sternförmige und die vermaschte Topologie. Bei der sternförmigen Topologie wird eine Verbindung zwischen Fernsehturm und Taxizentrale hergestellt und vom Fernsehturm aus werden die anderen Funkmasten angeschlossen. Die andere, teurere Möglichkeit besteht in der Vermaschung der Strecken. Das bedeutet, dass alle Stationen untereinander mit jeder anderen verbunden sind. Das erhöht einerseits die Ausfallsicherheit, andererseits aber auch die Infrastrukturund laufenden Kosten.

Um einen Mix aus Ausfallsicherheit in Verbindung mit möglichst geringen Kosten zu erstellen, haben wir uns für eine Kombination entschieden: In der Stadt werden die Sendeanlagen vermascht, außerorts werden die Anlagen sternförmig an den Fernsehturm angebunden. So ist für die Stadt eine hohe Ausfallsicherheit gewährleistet und die Kosten für die Anbindung der Sendeanlagen außerorts halten sich in Grenzen. Die Vermaschung betrifft die Standorte Fernsehturm, Pyramide und Müggelturm, die Sternanbindung die Standorte Schäferberg und Hennigsdorf.

Zur Errichtung der eigentlichen Funkanlage kommen pro Standort die zusätzlichen Infrastrukturbau- 
maßnahmen hinzu. Dazu zählen die Erdung des Masts, die Technikschränke, Licht, Sicherungen, Manschetten, Kabel, Leitern usw.

\subsubsection{Funkzentrale}

Die Zentrale ist mit einer Telekommunikationsanlage an das öffentliche Telefonnetz angeschlossen. Hinzu kommt eine Internetverbindung für elektronische Zahlungsvorgänge. Diese wird aus einer $4 \mathrm{MBit}$-Standleitung bestehen.

Die Funkanlage ist mit dem Masterswitch entweder über eine Standleitung verbunden, wenn der Switch weiter entfernt ist, oder direkt, wenn er sich im gleichen Gebäude befindet.

Zur Zentrale gehören auch die PCs an den Arbeitsplätzen der Mitarbeiter. Die Zentrale muss allerdings nur modernisiert und nicht neu gebaut werden. Das senkt die anfallenden Kosten erheblich. Uns ist nicht bekannt, ob die im Moment genutzte Software für die Disponierung der Fahrzeuge unabhängig vom verwendeten Funksystem ist oder ebenfalls ersetzt werden muss.

\section{Markt}

\subsection{Marktpotenzial}

Die Anwendungsgebiete und -möglichkeiten für digitale Bündelfunknetze in Deutschland sind sehr vielfältig und verbreitet. Speziell für das bearbeitete Projekt, die Ausrüstung einer Taxizentrale in Berlin, kann das erstellte Konzept ohne größere Umstände auch an andere Taxiunternehmen in ganz Deutschland vertrieben werden. Dabei würde jeweils lediglich eine Änderung der Netzinfrastruktur anfallen. So ist z. B. immer eine an die geografischen Gegebenheiten angepasste Funknetzplanung durchzuführen oder bei geringerer Zahl der zu versorgenden Fahrzeuge eine Reduzierung der zu betreibenden Kanäle zu berücksichtigen.

Bisher wird deutschlandweit von Taxiunternehmen fast ausschließlich analoger Bündelfunk oder vereinzelt auch noch Betriebsfunk zur Verständigung genutzt. In Berlin z. B. ist ein Konkurrent von Taxi Berlin zu nennen: »City-Funk GmbH Berlin «(derzeit ca. 300 Taxen). Diese setzen seit 1997 im Bereich des Datenfunks fünf eigene Betriebsfunkkanäle zur Netzabdeckung des Berliner Raums ein.

Eine Auf- bzw. Umrüstung auf digitalen Bündelfunk mit TETRA wäre in vielerlei Hinsicht sinnvoll. Einerseits können mittels dieser Technologie die analogen Störungen und Überlastungen des alten Netzes behoben, andererseits durch größere Datenübertragungsraten zusätzliche Dienste eingebunden werden. So wären Applikationen, wie z. B. Videostreams von Sicherheitskameras im Inneren der Fahrzeuge, denkbar (bei den zunehmenden Übergriffen auf Taxifahrer sicherlich eine sinnvolle Präventivmaßnahme). Neben der Übertragung von GPSDaten oder auch FCD (Floating Car Data) könnten auch Daten zum Infotainment des Passagiers zur Verfügung gestellt werden (ähnlich den Monitoren in U-Bahnen oder einer Internetverbindung), um die Attraktivität des Taxiunternehmens zu steigern.

Die Einführung von TETRA wird auch durch die aktuelle wirtschaftliche Lage der Anbieter analoger Bündelfunknetze begünstigt. So musste im vergangenen November das ohnehin schon angeschlagene Kölner Mobilfunkunternehmen »Dolphin Telecom $\mathrm{GmbH}$ « endgültig Insolvenz anmelden und damit seine Marktführerschaft von ca. $95 \%$ aufgeben. Laut Eigenangaben ist Dolphin mit über 8.000 Kunden und 75.000 betriebenen Geräten der größte Anbieter im analogen Bündelfunk. Kunden sind unter anderem Thyssen, Krupp, Daimler, Flughäfen, Stadtreinigungen, Rettungsdienste und Speditionen. Auch Taxi- und Mietwagenfirmen nehmen die Dienste von Dolphin in Anspruch. Derzeit laufen intensive Gespräche, um lukrative Teilnetze auszugliedern, zu verkaufen oder anderweitig weiter zu betreiben. Laut Auskunft der Dolphin-Geschäftsführung gibt es allein für die Region Berlin-Brandenburg fünf Interessenten.

Neben dem Geschäft für Taxizentralen ist das diskutierte Konzept auch für Anwendungsgebiete ähnlicher Aufgaben- und Anforderungsstruktur anwendbar. Hierzu zählen in erster Linie alle BOS-Dienste (Feuerwehr, Rettungsdienste, Polizei etc.), darüber hinaus aber auch private Anbieter von Krankentransporten, regionale Speditionen, Busunternehmen und Eigentümer mit großen Firmengrundstücken (z. B. Funkversorgung für das Braunkohlekraftwerk »Schwarze Pumpe« in Sachsen). Die grundsätzliche Lösung und Umsetzung bleibt in der Regel nahezu gleich: Aufbau einer passenden Netzstruktur, Aufbau einer Leitzentrale, Versorgung mit passenden Endgeräten, Schulung der Mitarbeiter und ggf. Wartung/Betrieb des Systems bzw. bestimmter Systemteile. Aufwendigere Anpassungen fallen normalerweise nur bei der Indoor-Vernetzung größerer Gebäude (Fabriken etc.) an.

Um die Nutzung eines bestehenden TETRA-Netzes kostengünstiger für den Nutzer zu gestalten, kommt die Mitnutzung durch einen anderen Kunden in Frage. So wird der Betrieb für beide Nutzer günstiger, da sich die laufenden Kosten für die Netznutzung aufteilen lassen. Dieses Beispiel ließe sich z. B. für die Ausrüstung von Taxi Berlin nutzen. Kommt ein weiteres Taxiunternehmen/ Spedition/Krankentransportunternehmen hinzu, ließen sich die Kosten je nach Nutzungsanteil aufteilen.

\subsection{Wettbewerber}

Neben adKor gibt es mehrere Mitbewerber, die ebenfalls TETRA-Lösungen anbieten. Im Folgenden sollen einige dieser Unternehmen kurz vorgestellt werden:

\subsubsection{Motorola}

Motorola ist ein international führendes Fortune-100Kommunikationsunternehmen, das mit Seamless Mobility-Produkten und -Lösungen nahtlose Mobilität über Breitband, eingebettete Systeme und drahtlose Netzwerke hinweg ermöglicht. In Deutschland ist das Unternehmen durch die Motorola $\mathrm{GmbH}$ präsent. $\mathrm{Zu}$ ihr zählen die Bereiche Mobiltelefone (Personal Devices), 
Mobilfunkausrüstung (Networks), Regierungs- und Geschäftskunden (Government and Enterprise) sowie Breitband (Connected Home). Die Gesellschaft erzielte 2004 mit über 2.500 Mitarbeitern einen Umsatz von rund 4,3 Milliarden Euro. Der Hauptsitz der Motorola GmbH ist in Taunusstein. Weitere Niederlassungen befinden sich in Berlin, Flensburg und München.

Motorola trug wesentlich zur Entwicklung des TETRA-Standards bei. Es war von Anfang an in den Gremien des ETSI (Europäisches Institut für Telekommunikation und Standardisierung) vertreten und zählt zu den Gründungsmitgliedern des TETRA Memorandum of Understanding, einer Vereinigung von Mobilfunkherstellern und Anwendern, die sich der Einführung des TETRA-Standards verpflichtet haben.

Motorola kann weltweit auf über 200 TETRA-Aufträge in mehr als 50 Ländern verweisen (Stand November 2005). Es stellt Betreiberkonzepte, Infrastruktur und Endgeräte zur Verfügung. Im November 2000 gewann Motorola die Ausschreibung für das Pilotprojekt Aachen (das erste deutsche TETRA-Test-Projekt im Realbetrieb). Das Pilotprojekt wurde im Jahre 2003 so erfolgreich abgeschlossen, dass es auch weiterhin in Betrieb ist. Zusammen mit T-Systems hat sich Motorola um den Ausbau/Umbau eines deutschlandweiten TETRA-Netzes beworben.

\subsubsection{Frequentis}

Frequentis ist ein österreichisches Unternehmen, welches Kommunikations- und Informationssysteme für sicherheitskritische Bereiche entwickelt. Die Kernkompetenz des Unternehmens liegt im Bereich der Sprachvermittlungssysteme für die Flugsicherung (Weltmarktführer mit einem Marktanteil von $30 \%)$. Weitere Geschäftsbereiche liegen in der Anwendung von Command \& Control-Systemen (Rettung, Polizei, Feuerwehr, Schifffahrt), im TETRA-Mobilfunk sowie bei Eisenbahnen und im öffentlichen Verkehr.

Das Unternehmen beschäftigt weltweit 587 Mitarbeiter (Stand 2004). Im Jahr 2004 wurde ein Umsatz von 92,9 Millionen Euro erwirtschaftet.

Auch Frequentis ist Mitglied im TETRA Memorandum of Understanding und betreibt mit der 3T Communications AG eine auf TETRA spezialisierte Tochterfirma. Das Frequentis Konsortium konnte seit 2002 mehr als 100 TETRA-Lösungen weltweit vertreiben und kooperiert dabei auch mit Motorola, indem es deren Verkaufskanäle nutzt.

\subsubsection{ATS Elektronik GmbH}

ATS ist ein kleines, mittelständiges Unternehmen, welches Hardware, Systemlösungen und Dienstleistungen im Kommunikations- und Sicherheitsbereich anbietet. Das Unternehmen beschäftigt ca. 20 Mitarbeiter und hat seinen Firmensitz in Hannover. ATS ist ein autorisierter Vertriebs- und Servicepartner der Motorola GmbH Geschäftsbereich Funk (Betriebs- und Bündelfunkendgeräte, Ersatzteil und Reparaturservice, Funk-Systemlösungen).

ATS bietet neben herkömmlicher GSM- und analoger Bündelfunktechnologie auch TETRA an. Dabei greift die
Firma hauptsächlich auf Entwicklungen und Know-how der Kooperationspartner Motorola und T-Mobile zurück. Über bisherige TETRA-Projekte lassen sich nur wenige Aussagen finden.

ATS ist ein Unternehmen, das sich in Größe und Struktur mit adKor vergleichen lässt, aber durch seine Kooperation mit Motorola und T-Mobile zusätzliche Vorteile bezüglich Erwerb und Nutzung von Technologien und Vertriebskanälen besitzt.

\subsubsection{Wettbewerbervergleich}

Die größten Konkurrenten von adKor sind Motorola und Frequentis. Durch ihre internationale, langjährige Erfahrung in einer Vielzahl von Projekten und durch die Entwicklung von eigenen Systemlösungen besitzen diese Firmen einen strategischen und finanziellen Vorteil gegenüber adKor.

Um diese Vorteile der Konkurrenten wettzumachen, kann sich adKor am Markt mit der Preiskomponente für Systemlösungen sowie seinen Serviceleistungen und ggf. mit einem Standortvorteil hervorheben.

\section{Marketing}

\subsection{Ziele}

Wie unter Punkt 5 beschrieben, besteht für adKor das größte Marktpotenzial in der flächendeckenden, deutschlandweiten Abdeckung der Taxiunternehmen mit digitalem Bündelfunk. Ziel des Marketings ist es folglich, diese Unternehmen von den Vorteilen der TETRA-Technologie gegenüber den bisher verwendeten Kommunikationstechnologien zu überzeugen und darüber eine möglichst große Marktabdeckung zu erreichen. Neben dem Aufbau der Netze besteht ein weiteres Ziel in nachhaltigen Gewinneinnahmen durch den Abschluss von Betriebs- und Wartungsverträgen für die Netze und/oder die Zentralen.

\subsection{Preispositionierung}

Der Preis für die angebotene Systemlösung ist die wichtigste Herausstellung von adKor gegenüber seinen Mitbewerbern.

Als Basis für die Preiskalkulation dient die Angabe der bisherigen Kosten, die bei Taxi Berlin pro Fahrer pro Monat in Höhe von 130 Euro anfallen. Ziel der neuen Technik soll eine Kosteneinsparung für die Fahrer sein. Kalkuliert wurden daher monatliche Kosten von ca. 70 Euro. Die einmaligen Einbaukosten für ein neues Funkgerät betragen pro Fahrzeug inkl. Montage ca. 950 Euro (Details unter Punkt 7.3).

Die Preisgestaltung richtet sich stark nach den laufenden Kosten der Netzinfrastruktur. In ländlichen bzw. dünn besiedelten Gebieten sind die Kosten pro Fahrer, bezogen auf die Infrastruktur, aus diesem Grund höher als in der Stadt. Für die nötige Funkversorgung ist mehr bzw. aufwendigere Technik notwendig. Diese höheren Kosten müssen auf weniger Fahrer aufgeteilt werden. So 
entsteht ein finanzieller Mehraufwand bzw. eine längere Amortisationszeit für die Anlage.

\subsection{Vertrieb und Werbung}

Da der Vertrieb praktisch nur Business-to-Business erfolgt, kommt eine herkömmliche breit angelegte Marketingstrategie (z. B. Fernseh-/Radiowerbung, Plakate, etc.) nicht in Frage. Vielmehr wird der Vertrieb über klassische Akquise (speziell Kaltakquise) realisiert werden, ein insbesondere für B2B-Geschäfte gängiges Marketinginstrument. Die Erfolgsquote für einen Geschäftsabschluss im Rahmen der Kaltakquisition wird in der Literatur zwischen drei und 20 Prozent geschätzt. Diese Quote hängt in erster Linie von Faktoren wie dem Ansehen der Firma, der Qualität des Produktes und erfolgreichen Referenzprojekten und -partnern ab. Eine Steigerung dieser Quote sollte also durch das Gewinnen imageträchtiger Partner (z. B. Kooperation mit Markenherstellern) und prestigeträchtiger Projekte (z. B. die Versorgung aller Taxizentralen/-unternehmen in ganz Berlin) möglich sein. adKor hat auf diesem Gebiet den Vorteil, etliche Referenzen vorweisen zu können (siehe Homepage).

Bei der Kundenakquisition unterscheidet man nach Push-Methoden (aktives Werben) und Pull-Methoden (passives Werben), die beide zum Einsatz kommen sollen. Der Schwerpunkt wird zunächst auf den Push-Methoden liegen, da zunächst die Akzeptanz für den Wechsel von analogem zu digitalem Bündelfunk steigen und das Geschäftsmodell bekannt und populär gemacht werden muss. Ein aktives Herantreten an den Kunden ist dabei unerlässlich.

Inhaltlich soll, mit den Vorteilen von TETRA gegenüber dem analogen Bündelfunk wie oben beschrieben geworben werden. Argumente und Vorteile sind unter anderem:

- Abhörsicherheit: höchste Übertragungssicherheit durch Ende-zu-Ende-Verschlüsselung aller Daten.

- Gruppenkommunikation mit dynamischer Gruppenbildung.

- Einwahlmöglichkeit in andere Kommunikationsnetze wie GSM oder das öffentliche Vermittlungsnetz.

- Direct-mode-Kommunikation zwischen Endgeräten, sogar bei Netzausfall.

- Datenkommunikation bis hin zur IP-Integration.

- Notrufverbindungen mit optionaler Zwangsfreischaltung von Kanälen.

- Einzelverbindung mit Duplex-Möglichkeit.

- Frequenzsparender Einsatz durch Gleichwellen-Technologie.

- Einfache, PC-gestützte Netz- und Gruppenverwaltung.

- Hohe Datenübertragungsraten (Breitband-TETRA (TETRA 2) mit bis zu $400 \mathrm{kBit} / \mathrm{sec}$ ).

- Gleichzeitige Übertragung von Sprache und Daten.

- Zuverlässiger, ungestörter Zellwechsel ohne Kommunikationsunterbrechung.

- Unterstützung vielfältige Anwendungen mittels offen definierter Schnittstellen.

\section{Finanzplanung}

\subsection{Standortplanung}

Um den Raum Berlin flächendeckend mit dem digitalen Bündelfunksystem auszustatten, sind mindestens fünf Standorte notwendig. Wie oben beschrieben, werden die BS-Standorte voraussichtlich Müggelturm, Pyramide, Fernsehturm, Schäferberg und ein $300 \mathrm{~m}$ hoher Mast in Hennigsdorf sein.

Für alle Standorte fallen zunächst Installationskosten an. Eine detaillierte Kostenplanung für die einzelnen Standorte wurde durchgeführt. Neben der Standortplanung und -akquise gehören dazu die TETRA-Hardware (Sektorantennen), die untereinander bestehenden Linkverbindungen (Richtfunklink, 2 MBit-Link) sowie die Kosten für Aufbau und Installation (Civil Works). Unter Civil Works haben wir die Kosten für die Bodenplatte, Masten, Kabel, Manschetten, Schaltschränke usw. zusammengefasst. Die Kosten belaufen sich auf 60.000 bis 80.000 Euro pro Standort. Die Gesamtkosten der fünf Standorte belaufen sich auf 355.620 Euro.

Neben den einmaligen Installationskosten sind die laufenden Kosten kalkuliert worden. Um einen Vergleich zwischen allen Werten zu ermöglichen, wurden alle laufenden Kosten auf einen Monat umgelegt. Versicherungen, die monatlich anfallenden Gebühren für die Richtfunkverbindungen und 2 MBit-Standleitungen sowie die Energiekosten sind hier die Hauptaspekte. Für alle fünf Sendeanlagen fallen in unserer Kalkulation monatliche Kosten in Höhe von 10.915 Euro an.

Abschließend wurden die EMV-Untersuchung sowie die Anmeldegebühr für die TETRA-Nutzung kalkuliert. Es ergeben sich folgende Eckdaten für die fünf Standorte, auf denen unsere Investitionsplanung basiert:

- Installationskosten: 358.470 Euro

- Betriebskosten monatlich: 10.915 Euro

\subsection{Planung der Leitstelle (Zentrale)}

Die Kosten der Leitstelle setzen sich aus den fixen Kosten für die Einrichtung/Renovierung der alten Funkzentrale und den variablen Kosten, die pro Monat anfallen, zusammen.

Wir fassen die fixen Kosten in drei Punkten zusammen: 1. Neueinrichtung der Leitstelle mit Bürotechnik: Dazu zählen die Renovierung der Leitstelle und neue Büromöbel. Dieser Posten beträgt nach unserer Kalkulation 30.000 Euro.

2. Ausstattung der Zentrale mit 10 Arbeitsplätzen: Das umfasst jeweils einen PC und die benötigte Software. Dieser Posten umfasst 20.000 Euro.

3. Einrichtung der 2 MBit-Standleitung bei der T-Com. Diese berechnen wir mit 3.500 Euro.

So summieren sich die einmaligen Installationskosten auf einen Betrag von 53.500 Euro.

Die monatlich anfallenden Kosten umfassen Miete, Strom, Gas, Wasser, Personal- und Telefonkosten und die monatliche Gebühr für die Standleitung. Diese summieren sich nach unserer Annahme auf ca. 49.500 Euro. 
Ausgehend von 20 Mitarbeitern und einem Vorgesetzten sieht das Modell wie folgt aus: 20 Mitarbeiter arbeiten in einem Dreischichtsystem, wobei je acht Angestellte in der Früh- und Spätschicht arbeiten und vier in der Nachtschicht. Pro Mitarbeiter wird mit einem Bruttolohn von 2.000 Euro gerechnet. Der Vorgesetzte fließt mit 3.500 Euro in die Planung ein. Summiert ergeben sich Personalkosten von 43.500 Euro. Die einmalige Schulung aller 20 Mitarbeitern kalkulieren wir mit einmalig 5.000 Euro.

\subsection{Installationskosten Taxi}

Zusätzlich haben wir die Kosten für die Anschaffung neuer Funkgeräte berechnet. Ausgehend von 1.600 angeschlossenen Taxis und Gerätekosten von ca. 800 Euro inkl. Mengenrabatt sowie Einbaukosten in Höhe von ca. 150 Euro pro Fahrzeug summieren sich diese auf insgesamt 1.280.000 Euro. Diese sind von den Taxifahrern anteilig selbst zu tragen. Durch die Einrichtung eines Pools für die Geräte und den Einbau können Mengenrabatte ausgehandelt werden. Die Kosten werden jedoch nicht von adKor oder Taxifunk übernommen bzw. vorgestreckt und gehen daher nicht weiter in die Kalkulation ein.

\subsection{Gewinn und Verlust Rechnung}

In den ersten drei Monaten fallen lediglich die Installationskosten der Zentrale und der fünf Sendeanlagen an. Zusätzlich werden hier die Abschreibungen und Zinsen zur Kredittilgung berücksichtigt. In den Umsätzen werden die Einnahmen von Taxi Berlin durch die bei ihnen registrierten Taxifahrer berücksichtigt. In Berlin sind derzeit ca. 1.600 Taxis bei Taxi Berlin registriert. Diese zahlen derzeit ca. 130 Euro pro Monat, um die Vermittlung durch Taxi Berlin nutzen zu können. Damit das TETRA-System gut im derzeitigen Markt positioniert werden kann, sollte hier mit einer monatlichen Pauschale von 70 Euro kalkuliert werden. Die Einnahmen würden sich somit auf max. 112.000 Euro (bei 1.600 registrierten Taxis) belaufen. Anhand des kumulierten Betriebsergebnisses wird der maximale Kapitalbedarf berechnet. Dieser beläuft sich für das komplette System (Netzwerk und Zentrale) auf 462.800 Euro.

\subsection{Gesamtkalkulation}

Der Investitionsbedarf ist unterteilt in die Kosten für die Zentrale und die Netzinfrastruktur. Diese summieren sich auf 416.970 Euro. Die monatlichen Kosten der Anlage gliedern sich in die laufenden Kosten für die 5 Funkstandorte und die Zentrale. Diese summieren sich auf 60.415 Euro monatlich.

\section{Geschäftsszenarien}

Es wurden drei Szenarien gerechnet.

\subsection{Szenario I: Netz in Besitz der Firma adKor}

Im ersten Szenario besteht eine grundlegende unternehmerische Trennung zwischen der Zentrale und der eigentlichen Netzinfrastruktur. Die Firma adKor stellt die gesamte TETRA-Netzinfrastruktur sowie die Verbindungen zwischen den Sendeanlagen (2 MBit-Link, RiFu) zur Verfügung. Die Installationskosten belaufen sich auf 355.620 Euro. Dieser Betrag fällt für alle fünf Sendeanlagen, den untereinander bestehenden Links sowie der Anbindung an die Leitstelle an. Taxi Berlin übernimmt parallel die Umrüstung und Anpassung der Leitstelle. In unseren Berechnungen gehen wir von einem Installationszeitraum von drei Monaten aus.

Die Installationskosten in Höhe 355.620 Euro müssen von der Firma adKor durch eine adäquate Finanzierung getragen werden. Danach soll die gesamte Infrastruktur des Netzes über einen Zeitraum von 5 Jahren rückfinanziert werden.

Unsere Finanzplanung enthält eine Marge von 10 Prozent auf die monatlich fällige Rate, damit kommen wir auf eine Gesamtrate von 22.376,20 Euro. Dieser Betrag muss von Taxi Berlin monatlich getragen werden.

Da die Firma adKor im Besitz des Netzes ist, besteht in diesem Szenario die Möglichkeit, dass weitere Teilnehmer das Netz nutzen, denn TETRA bietet die Möglichkeit unterschiedliche Nutzergruppen zu verwalten. Durch diese Maßnahme könnten die anfallenden Netzbetriebskosten auf mehrere Nutzer aufgeteilt werden, so dass die Netznutzung für alle Nutzer preisgünstiger wird. Die Abrechnung würde sich nach der angefallenen Netznutzung richten.

\subsection{Szenario II: Komplettverkauf an Taxi Berlin}

Das zweite mögliche Szenario ist der komplette Verkauf der Anlage an Taxi Berlin. Dazu zählen neben der Zentrale auch die Netzinfrastruktur und das eigentliche Funknetz. Die Firma adKor bliebe als regelmäßige Einnahme ein Servicevertrag für die Reparatur und Instandhaltung des Netzes.

Dieses Szenario wird von Seiten der Firma adKor nicht angestrebt, da sie an einer langfristigen Geschäftsbeziehung und damit langfristigen Einnahmen interessiert ist. Die Kaufsumme der Anlage liegt bei 458.667 Euro. Das umfasst die Kosten der Anlage von 416.970 Euro und eine Gewinnmarge von 10 Prozent.

\subsection{Szenario III: Zentrale und Netz in Besitz von adKor}

Das dritte Szenario stellt den Fall dar, dass die komplette Anlage im Besitz von adKor verbleibt und nur an Taxi Berlin vermietet wird. Bei dieser Kalkulation müssen die Infrastruktur- und die laufenden Kosten der Anlage 
und Zentrale in Form einer monatlichen Gebühr (Miete) durch Taxi Berlin finanziert werden.

Der Zeitraum für die Refinanzierung wird mit fünf Jahren angenommen. Die Installationskosten für Netz und Zentrale werden auf fünf Jahre à zwölf Monate aufgeteilt. Dadurch ergibt sich der zu entrichtende Beitrag zu den einmaligen Kosten. Die laufenden Kosten für Zinsen, die monatlichen Kosten der fünf Standorte sowie die Kosten der Zentrale (Miete, Strom, Gas, Wasser, Telefon, Standleitung) werden hinzuaddiert. Dazu kommt noch eine geringe Marge für adKor. Das ergibt eine monatliche Mietgebühr für das Funksystem in Höhe von 30.507,03 Euro.

Dieses Szenario wäre ein durchaus akzeptables Geschäft für adKor, obwohl die Firma auch die Kosten für die Instandhaltung der Zentrale übernehmen müsste.

\subsection{Schlussfolgerung aus den Szenarien}

adKor bevorzugte das erste der drei Geschäftsmodelle. Die Firma wäre nicht für den Betrieb der Zentrale zuständig, sondern könnte sich auf ihr Kerngeschäft, den Mobilfunk, beschränken. Dazu kämen stetige Einnahmen durch die monatliche Netznutzungsgebühr. Sollte sich, wie im Kapitel Marktpotenzial angesprochen, ein weiterer Nutzer für das Netz finden lassen, würden die Kosten für Taxi Berlin sinken und das Modell wäre noch attraktiver.

\section{Fazit}

Im Laufe dieser Studie wurden die Vorteile des digitalen gegenüber dem analogen Bündelfunk deutlich. Durch die Klassifizierung von einzelnen Nutzergruppen ist es möglich, die gesamte Infrastruktur auch anderen zur Verfügung zu stellen. Derzeit wird in Taxi-Betrieben und anderen Unternehmen (Nahverkehrsbetriebe, Hauskrankenpflege etc.) hauptsächlich der analoge Bündelfunk eingesetzt. Das TETRA-System für Taxi Berlin kann als Referenzsystem für die Anbindung von kleinen und mittleren Unternehmen an das digitale Bündelfunknetz genutzt werden. Damit würde das System selbst zum Marketinginstrument und könnte weitere Firmen dazu bewegen, sich diesem System anzuschließen. Damit könnte sich die Firma adKor $\mathrm{GmbH}$ als bundesweiter Anbieter von TETRA-Bündelfunktechnik etablieren und einen hohen Marktanteil sichern.

Das dargestellte Szenario I ist das für adKor langfristig attraktivste Modell. Durch die Vermietung und Wartung der Netzinfrastruktur lassen sich hier langfristig konstante Einnahmen generieren.

Die verwendeten Zahlen waren aus Wettbewerbsgründen synthetische Zahlen. Der Wert der Studienarbeit besteht in erster Linie darin, als Rahmen und Basis für die Erstellung eines realistischen Angebots seitens der Firma adKor an Taxi Berlin und ggf. darüber hinaus in anderen Bündelfunklizenzgebieten zu dienen.

So kam der Auftraggeber zwar nicht bei Taxi Berlin zum Zuge, dafür aber kurze Zeit später in Hamburg mit demselben von uns generierten Geschäftsmodell zum Erwerb des Bündelfunknetzes Nord sowie in Hannover zum Erwerb des dortigen Bündelfunknetzes.

\section{Danksagung}

Die Ausarbeitung ist eine Kurzfassung des Abschlussberichts zum Forschungsvorhaben: »Erstellung eines Geschäftsmodells zur Ausrüstung von Taxi Berlin mit einem digitalen Bündelfunksystem «, Projekt Nr. 115 112 01, im Auftrag der Firma adKor GmbH, Wildau und wurde im Rahmen des Wettbewerbs »Effiziente Kooperationen zwischen Hochschulen und Unternehmen/Verwaltungen « aus Anlass der Veranstaltungsreihe der TFH Wildau »15 Jahre zuverlässiger und erfolgreicher Partner der Wirtschaft und Verwaltung «am 27. April 2007 mit einer Ehrenurkunde ausgezeichnet. Die Autoren danken der TFH Wildau und der Firma adKor für Auftrag, Durchführungsmöglichkeit und Unterstützung.

\section{Internetquellen}

Nachrichtenartikel über die Insolvenz der Dolphin Telecom vom 04.11.2005, www.taxizeitschrift.de

Startseite der europäischen Taximessen, Zugriff 19.01.2006, www.eurotaximesse.de

Firmenhomepage der adKor GmbH, Zugriff 17.01.2006, www.adkor.de

Firmenhomepage der Frequentis AG, Zugriff 24.01.2006, www.frequentis.com

Firmenhomepage der Motorola Inc., Zugriff 24.01.2006, www.motorola.com

Firmenhomepage der ATS Elektronik GmbH-Alarmanlagen, Zugriff 24.01.2006, www.atsonline.de

Firmenhomepage der 3T Communications AG, Zugriff 24.01.2006, www.3t-ag.com

\section{Autoren}

Prof. Dr.-Ing. Bernd Eylert

Technische Fachhochschule Wildau

Tel. +49 3375 508-120

bernhard.eylert@tfh-wildau.de

Patrik Groh, M. Eng.

Ottomar-Geschke-Straße 79

12555 Berlin

Eike Kräft, M. Eng.

Waldstraße 94

12527 Berlin

Thomas Weidner, M. Eng.

Erwin-Bock-Straße 4

12559 Berlin 